\title{
A Case of Hypercalcemia in an Immunocompetent Patient with Disseminated Mycobacterium marinum Infection with a Rain Barrel as the Most Likely Primary Source
}

\author{
Wendy Uijtendaal $^{1}$, Rima Yohanna ${ }^{2}$, Folkert W Visser ${ }^{1}$, Paul M Ossenkoppele ${ }^{3}$, Dorine LJ Hess ${ }^{4}$, Dennis Boumans ${ }^{2}$ \\ ${ }^{1}$ Department of Internal Medicine, Ziekenhuisgroep Twente, Almelo, The Netherlands \\ ${ }^{2}$ Department of Rheumatology, Ziekenhuisgroep Twente, Almelo, The Netherlands \\ ${ }^{3}$ Department of Dermatology, Ziekenhuisgroep Twente, Almelo, The Netherlands \\ ${ }^{4}$ Department of Microbiology, Ziekenhuisgroep Twente, Almelo, The Netherlands
}

Received: 06/09/2021

Accepted: 05/10/2021

Published: $18 / 11 / 2021$

How to cite this article: Uijtendaal W, Yohanna R, Visser FW, Ossenkoppele PM, Hess DLJ, Boumans D. Disseminated Mycobacterium marinum infection with a rain barrel as the most likely primary source. EJCRIM 2021;8: doi:10.12890/2021_002864.

Conflicts of Interests: The authors declare there are no competing interests.

This article is licensed under a Commons Attribution Non-Commercial 4.0 License

\section{ABSTRACT}

Infection with Mycobacterium marinum is common in fish, and so human infection usually arises from contact with contaminated water or fish. A solitary papulonodular lesion on a finger or hand is the typical presentation. Disseminated infections are rare and mostly seen in immunocompromised patients.

We present a rare case of disseminated M. marinum infection presenting with polyarthritis, tenosynovitis, dactylitis, and (sub)cutaneous and intramuscular lesions in an immunocompetent patient. This case was complicated by hypercalcemia, renal failure and eventually death. A contaminated rain barrel was most likely the primary source of the infection.

\section{LEARNING POINTS}

- Given the similarities, it is key to differentiate Crohn's disease from intestinal tuberculosis as early as possible.

- Patients undergoing colonoscopy for possible Crohn's disease should have colonic biopsy samples sent for AFB culture.

- Consider investigations for intestinal tuberculosis in uncontrolled Crohn's disease where intestinal tuberculosis has not been worked up previously.

\section{KEYWORDS}

Mycobacterium marinum, hypercalcemia, immunocompetent, polyarthritis, polyarteritis nodosa

\section{INTRODUCTION}

Mycobacterium marinum is one of the $>150$ species of the genus Mycobacterium and together with M. ulcerans is the most closely related to $M$ tuberculosis. These two differ also from the other non-tuberculous mycobacteria (NTM) in being non-opportunistic pathogens. M. marinum disease is common in fish. Therefore, most people become infected through handling contaminated water or fish, giving it its nickname 'fish tank granuloma'. Infection with M. marinum has different clinical presentations, although $60 \%$ of patients will present with a solitary papulonodular lesion on a finger or hand. This is probably due to the optimal growth temperature of $30^{\circ} \mathrm{C}$. 
Some $20-40 \%$ of patients have deep infections like tenosynovitis (most frequent), osteomyelitis, arthritis and dactylitis. Systemic dissemination is exceptional and mostly reported in immunocompromised patients ${ }^{[1-3]}$.

\section{CASE DESCRIPTION}

An 86-year-old female patient with a medical history of hypertension, transient ischemic attack and chronic kidney disease, presented at the rheumatology outpatient clinic with extensor tenosynovitis of the left wrist, polyarthritis of MCP joints and the left wrist, and dactylitis of the second digit of the right hand. There were a few skin lesions: two small nodular lesions on the left arm and right leg and a larger crusty ulcer on the left thigh. Due to an elevated urate of $0.40 \mathrm{mmol} / \mathrm{I}$ and a possible urate crystal in the synovial fluid of the MCP joint, the patient was treated with colchicine, with a working diagnosis of polyarticular gout. Because of worsening of the arthritis, prednisolone was added without achieving any improvement. The prednisolone dosage was increased to $30 \mathrm{mg}$ a day when some existing nodular cutaneous lesions multiplied. The pathology report of a biopsy of these lesions showed evidence of cutaneous polyarteritis nodosa (cPAN). Eventually the patient was admitted to hospital due to progression of these lesions leading to widespread pustular papules and ulcers, including on the face but not on her back (Fig. 1A,B).
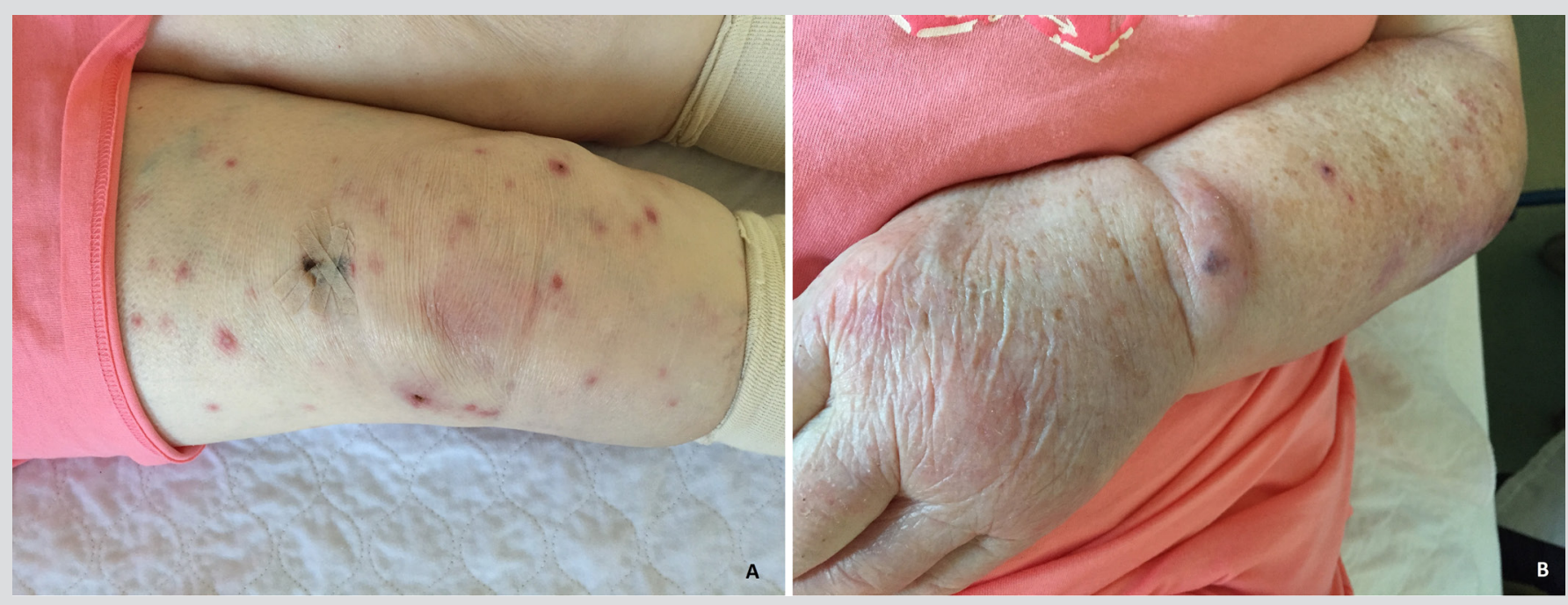

Figure 1. Skin lesions in our patient on the $(A)$ right leg and $(B)$ left wrist

Initial cultures of skin biopsies (taken 6 weeks before admission) and later cultures and PCR of synovial fluid, pustules and repeated skin biopsies were positive for M. marinum. Furthermore, Ziehl-Neelsen staining on skin biopsies was positive for acid-fast bacilli, the QuantiFERON-TB GOLD test was positive and a 18F-FDG PET/CT scan showed extensive cutaneous, subcutaneous and intramuscular (nodular) lesions, supporting disseminated disease (Fig. 2). The 18F-FDG PET/CT scan did not show any signs of malignancy.

These findings confirmed the diagnosis of disseminated M. marinum infection with polyarthritis, tenosynovitis, dactylitis, and extensive (sub) cutaneous and intramuscular lesions. The diagnosis of cPAN and gout was rejected. Interestingly, the patient did not swim and had never handled or touched an aquarium. However, she watered her garden with water from her rain barrel; testing of this water was positive for multiple species of Mycobacteria.

The patient began treatment with azithromycin, ethambutol and rifampicin. The prednisolone dosage was gradually tapered as the joint complaints improved. However, 8 weeks after starting treatment, the skin lesions had only improved slightly and also a new hypercalcemia (corrected calcium: $4.19 \mathrm{mmol} / \mathrm{l}$ ) with renal failure (creatinine $181 \mu \mathrm{mol} / \mathrm{L}$, eGFR $24 \mathrm{ml} / \mathrm{min}$ ) was noticed. The PTH level was low-normal (1.6 pmol/l), 25-OH-vitamin D and 1,25-(OH)2D3 were both high-normal (69 nmol/I and $142 \mathrm{pmol} / \mathrm{I}$ respectively), and the PTH-related peptide was low $(<0.3 \mathrm{pmol} / \mathrm{l})$. Despite successful initial treatment with hyperhydration, the hypercalcemia progressed within a week, however now with extensive confusion. Because of the lack of improvement in the previous months and progressive hypercalcemia with renal failure (creatinine $340 \mu \mathrm{mol} / \mathrm{L}$, eGFR $10 \mathrm{ml} / \mathrm{min}$ ), and on the request of the patient, we decided to stop further treatment and the patient died within a week. 


\section{DISCUSSION}

In this case, at first presentation in the outpatient clinic, there already was disseminated M. marinum infection given the skin lesions on the thigh, tenosynovitis, polyarthritis and dactylitis, and positive cultures and PCR with M. marinum. As the literature shows, this is an uncommon feature in immunocompetent people ${ }^{[1,3]}$. Our patient did not receive any immunosuppressive therapy and did not have hypogammaglobulinemia, hypocomplementemia, cryoglobulinemia, paraproteinemia or an underlying malignancy. As result of the prednisolone, the disseminated M. marinum infection, which was already present at first presentation, progressed, leading to extensive (sub) cutaneous and intramuscular lesions as shown by the 18F-FDG PET/CT scan (Fig. 2).

Another atypical finding in this case is the fact our patient did not swim and had never handled fish or an aquarium. She watered her garden from a rain barrel in which we later found several species of Mycobacteria, making it the most likely primary source of the infection.

The last atypical finding is the striking hypercalcemia. Hypercalcemia is not unusual in several granulomatous diseases like mycobacterial infections. The supposed mechanism is through endogenous production of $1,25-(\mathrm{OH}) 2 \mathrm{D} 3$ in disease-activated macrophages in granulomas $^{[4,5]}$. This process might be upregulated in the case of discontinuation of immunosuppressive therapy resulting in a rebound effect of interferon gamma on the granulomas. Two-thirds of skin biopsies in M. marinum infection show granulomas ${ }^{[1]}$. M. marinum disease is rarely disseminated, and there is only one case report on hypercalcemia in an (also immunocompetent) patient with disseminated $M$. marinum infection ${ }^{[4]}$. Our patient showed a high-normal 1,25-(OH)2D3 and developed hypercalcemia after withdrawal of the prednisolone, so the supposed mechanism might stand in our patient; nonetheless, the pathology report of the biopsies showed no evidence of granulomas, which could also be explained by sampling error. Also, other causes of the hypercalcemia such as hyperparathyroidism, vitamin D overuse or intoxication, malignant lymphoma, multiple myeloma or solid malignancies were excluded, making the M. marinum infection the most logical explanation. 


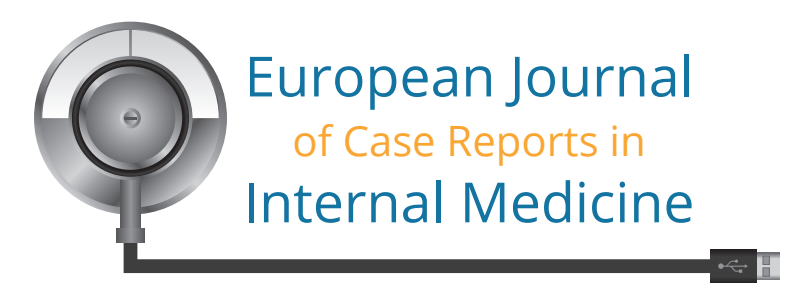

\section{REFERENCES}

Aubry A, Mougari F, Reibel F, Cambau E. Mycobacterium marinum. Microbiol Spectr 2017;5(2).

Johnson MG, Stout JE. Twenty-eight cases of Mycobacterium marinum infection: retrospective case series and literature review. Infection $2015 ; 43(6): 655-662$.

Holden IK, Kehrer M, Andersen AB, Wejse C, Svensson E, Johansen IS. Mycobacterium marinum infections in Denmark from 2004 to 2017: a retrospective study of incidence, patient characteristics, treatment regimens and outcome. Sci Rep 2018;8(1).

4. Nielsen CT, Andersen ÅB. Hypercalcemia and renal failure in a case of disseminated Mycobacterium marinum infection. Eur J Intern Med 2009;20(2):e29-31.

5. Abdulfattah O, Rahman EU, Shweta F, Datar P, Alnafoosi Z, Trauber D, et al. Severe hypercalcemia in a patient with extrapulmonary Mycobacterium abscessus: granuloma or immune reconstitution inflammatory syndrome? First case of Mycobacterium abscessus presenting as retroperitoneal lymphadenopathy with severe hypercalcemia: a case report and literature review. J Community Hosp Intern Med Perspect 2018;8(6):331-338. 